\author{
EVS29 Symposium \\ Montréal, Québec, Canada, June 19 - 22, 2016
}

\title{
Added Value for Electrified Vehicles through Enhanced Active Safety
}

\author{
Adithya Arikere $^{1}$, Anders Tysk ${ }^{1}$ \\ ${ }^{1}$ AAM (American Axle \& Manufacturing), Inc., \\ TTC - Trollhättan Technical Center, \\ Nohabgatan 18E, 46153 Trollhättan, Sweden \\ adithya.arikere@aam.com
}

\begin{abstract}
The possibility of using electric drives to improve safety is presented. Several opportunities were identified and discussed briefly. In particular, two use cases are analyzed in detail: first to accelerate the lead vehicle to prevent or mitigate a rear-end collision and second, to control speed for mitigation of collision risk with oncoming vehicles during evasive maneuvers. Significant safety benefit was estimated in both cases and hence, a strong opportunity is seen for adding safety related value to electrified vehicles at little extra cost.
\end{abstract}

Keywords: safety, electric drive, passenger car, vehicle performance

\section{Introduction}

\subsection{The push for electrification}

Over the past several decades, there has been a rapid increase in the mobility of the world population. A consequence of this is that there has been increasing $\mathrm{CO}_{2}$ emissions due to transportation. Coupled with an increasing awareness of climate change among the general public, this has resulted in increasing pressure on vehicle manufacturers to reduce $\mathrm{CO}_{2}$ emissions. Vehicle manufacturers have investigated numerous approaches to this including electrification, alternative fuel sources, downsizing, etc. Among these, vehicle electrification has emerged as the most promising option due to a variety of reasons. Studies investigating the capabilities of electrified drivetrains have shown that they have a strong potential to reduce greenhouse gases [1,2]. Additionally, public agencies have also been pushing for electrification with tax rebates for electrified vehicles. However, despite all these, electrified vehicles have not really captured the market.

A study done in 2009 by the International Energy Agency found that at the current rate of growth in electrified vehicle sales, we will miss the target sales numbers needed to successfully mitigate global warming in the year 2030 ([3], Fig. 1). A more recent study done in 2012 shows that even accounting for and assuming that countries adopt and stick to the new policies that have been announced for carbon reduction, we would still miss the electrified vehicle sales targets by a significant margin [4].

The low sales are largely due to customer reluctance to invest in electrified vehicles due to a number of reasons including high initial cost, range anxiety, lack of charging infrastructure, etc. It is clear therefore 


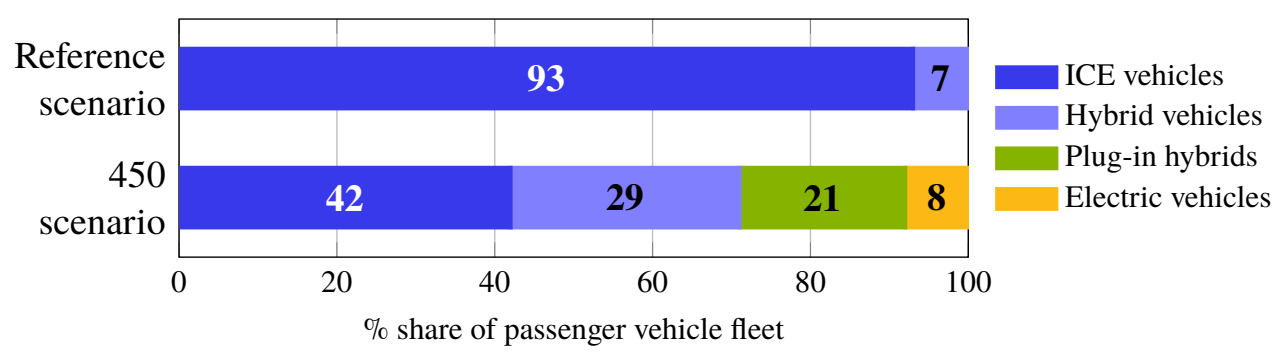

Fig. 1: Predicted actual (Reference scenario) vs needed share (450 scenario) of electrified vehicles in passenger vehicles sold for successfully mitigating global warming in the year 2030 (from [3])

that some form of added incentive is needed to make electrified vehicles attractive to consumers.

\subsection{The need for increased safety}

Another consequence of increasing mobility of the world population is that there is now also an increasing focus on traffic safety. As a result, numerous targets on reducing traffic fatalities and/or injuries have been set by various public agencies. For example, Sweden has the Vision Zero which aims to completely eliminate traffic fatalities by 2020 [5] and the UK has similar goals [6]. Several cities in the US have also adopted a similar Vision Zero goal [7, 8, 9, 10]. Similarly, the European Commission had a target to halve the number of traffic fatalities between 2001-10 which it failed to meet [11]. The EU has has now set another goal to halve the traffic fatalities between 2010-20 and current trends suggest that the EU will fail to meet this target as well [12].

In order to achieve these ambitious safety targets, future approaches to safety must not only consider, but exploit the new sensors, the information sources and the actuators that will be available in the cars of the future. Given that electrified vehicles seem to be needed and inevitable, one of major new actuators that will be available in the cars of tomorrow is: electric drives. This in turn leads us to the question (and the topic of this paper): can electric drives be used to improve traffic safety and if yes, how?

\section{The electric drive advantage}

To understand how electric drives can be used to improve safety, it is first important to understand how electric drives are different from traditional internal combustion (IC) engines in their capabilities. In this section, some of the advantages of electric drives over IC engines that are relevant for improving safety are reviewed.

\subsection{Expansion of vehicle dynamic capability}

Electric drives have much faster response when compared to traditional IC engines. Up to $200 \mathrm{~ms}$ might be required to just open the throttle actuator in some vehicles whereas electric drives can have response times as low as tens of milliseconds [13]. In addition, there are additional significant response times due to the dynamics of the drivetrain: inertia of the engine; spooling up of the turbocharger (if one is fitted); switching to the appropriate gear and so on. Electric drives on the other hand don't typically share these issues and can respond very quickly which is useful in emergency situations.

Since the peak performance of electric drives are, generally speaking, constrained by heat dissipation, they can be adapted to deliver higher performance than normal for brief periods which is useful in safety critical situations where fast, reliable and large magnitude actuator inputs are needed.

Since brake based interventions have to be limited in duration due to heating issues, they are usually only done at the last moments before a potential accident and are typically harsh. With electric drives on the other hand, due to the continuous operational capability, they can be used early on in safety critical situations to perform soft interventions. Additionally, their bi-directional nature means that errors can 
be quickly corrected for, leading to robust interventions. These features also make them suitable for continuous interventions in order to adapt the vehicle behavior to the situation or the driver leading to improved safety and comfort.

The ability of electric drives to apply tractive force on an axle quickly and reliably can be used in conjunction with differential braking to achieve the effect of torque vectoring. Torque vectoring has significant benefits over traditional differential braking for lateral and yaw control applications. First, due to the non-linear way in which the lateral and longitudinal tire forces add up, there is an inherent benefit in being able to distribute tire forces equitably. This can be achieved with torque vectoring since it can propel one of the wheels at an axle and brake the other at the same time. Secondly, this also enables torque vectoring to apply high yaw moments than differential braking alone possibly can. These in turn lead to a better tradeoff between global lateral force and yaw moment capabilities at the vehicle level as well. This is illustrated in Fig. 2 which shows the normalized global vehicle forces achievable, assuming the vehicle is in an on-limit cornering maneuver for a system with brakes alone (Fig. 2a) and a system with brakes and electric drives, i.e., torque vectoring (Fig. 2b). As can be seen, when applying the same yaw moment $\left(M_{z}^{g}=0.4\right)$ with differential braking, only $50 \%$ of the maximum lateral force capacity is retained $\left(F_{y}^{g}=0.5\right)$ whereas with torque vectoring, up to $70 \%$ is retained $\left(F_{y}^{g}=0.7\right)$ which represents a $40 \%$ improvement over the differential braking case.

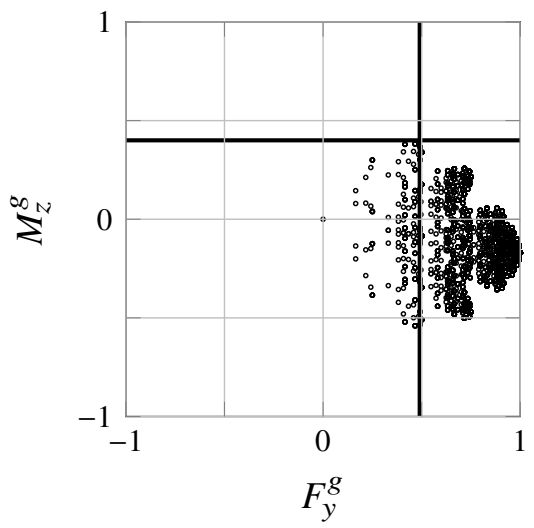

(a) Global force tradeoff with differential braking

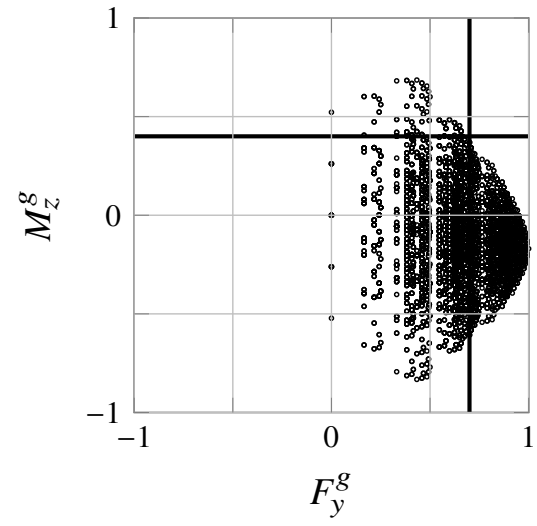

(b) Global force tradeoff with differential braking and electric drives

Fig. 2: Global vehicle forces

\subsection{Enhanced controllability}

The quick response time of electric drives allows for much finer control of slip in both braking and traction. For instance, in [14], the authors found a $7 \%$ reduction in braking distances using the electric drives for anti-lock braking actuation alone.

The bi-directional nature of electric drives allows for more robust control as it enables quick corrections to be performed using the same actuator. With brakes or engines on the other hand, the control will need to be conservative since any errors in control with either actuator will need to be corrected for with the other actuator. In addition, since both actuators have much slower and different response times, correcting for errors is not as easy as with electric drives either.

The ability of electric drives to operate continuously (as opposed to brakes) can be useful in performing in continuous interventions. Their quick response time and bi-directional nature only serve to make them more attractive for this application. For instance, in [15], the possibility of using electric torque vectoring for improving the yaw response of the vehicle was investigated and was found to be effective.

Another investigation done in [16] which investigated the potential of torque vectoring for lateral control 

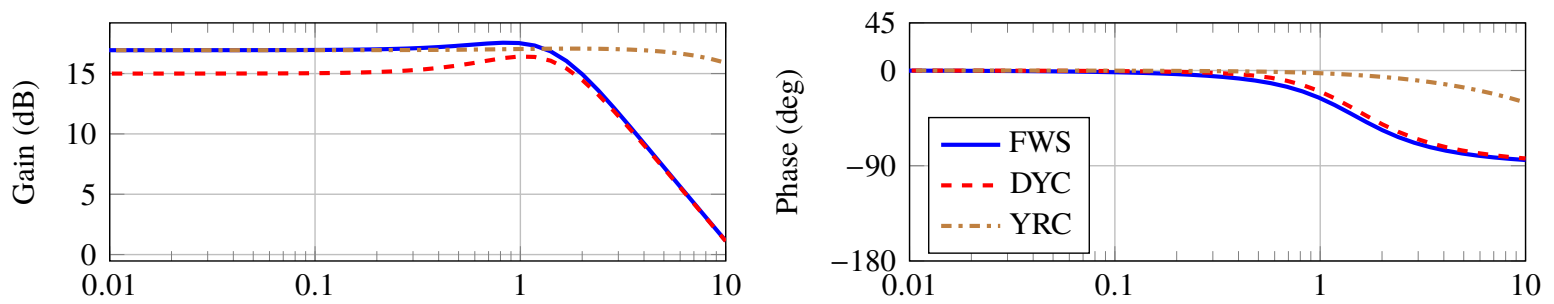

(a) Yaw rate response
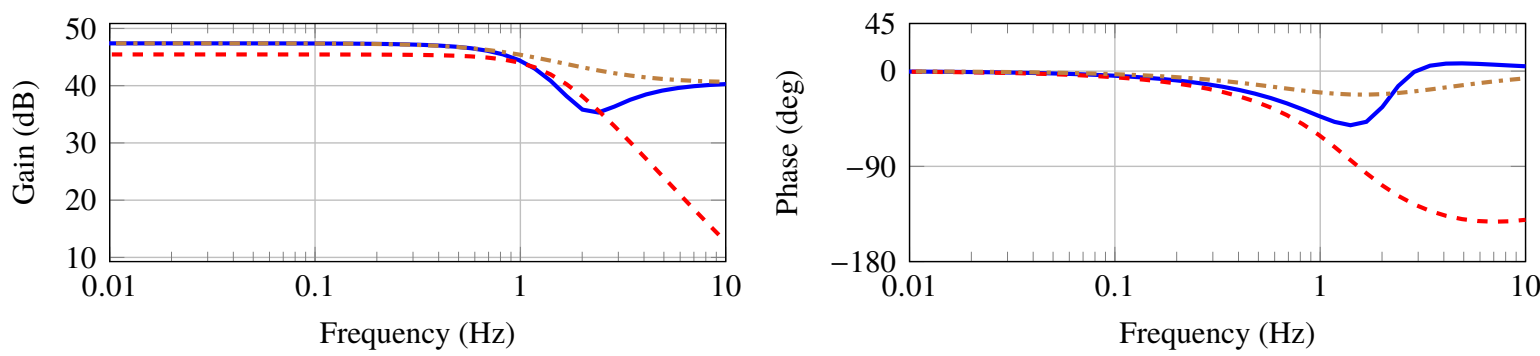

(b) Lateral acceleration response

Fig. 3: Frequency response plots for yaw rate and lateral acceleration using front wheel steering (FWS), torque vectoring used direct yaw control (DYC) and torque vectoring used for yaw response control (YRC). Vehicle speed is $120 \mathrm{~km} / \mathrm{h}$. (From [16])

and transient response improvement found that torque vectoring can achieve nearly the same performance or better as steering. It had some added benefit in terms of attenuation of high frequency disturbances in the lateral acceleration response characteristics (Fig. 3b). For transient response improvement on the other hand, torque vectoring was found to be very well suited as it significantly increased the bandwidth both for yaw rate and lateral acceleration response, reduced the phase shift and left the steady state response unchanged (Fig. 3a).

\section{Enhanced intervention opportunities}

The benefits associated with electric drives can be exploited to perform enhanced interventions in various accident scenarios to improve safety. Shown below are some of these use cases and how electric drives can be used to improve safety in each of them. In each of the following illustrations and scenarios, the vehicle labelled ' $\mathrm{H}$ ' represents the host vehicle, i.e., the vehicle with the electric drive and the vehicle labelled ' $\mathrm{B}$ ' represents the bullet vehicle or the obstacle that represents the threat.

\section{I $\longrightarrow$ क}

Fig. 4: Braking to avoid frontal collision

Shown in Fig. 4 is the frontal collision scenario wherein the host vehicle is the following vehicle and brakes automatically to avoid or mitigate the collision. As noted in $[17,18]$, typical hydraulic brakes can take up to $700 \mathrm{~ms}$ to build up to full brake pressure from zero when braking is done autonomously. This delay can be reduced when the electric drives are used for braking as well. Furthermore, as previously mentioned, the improved ABS and slip actuation that can be done using electric drives can result in a result in a braking distance reduction of up to $7 \%$. 


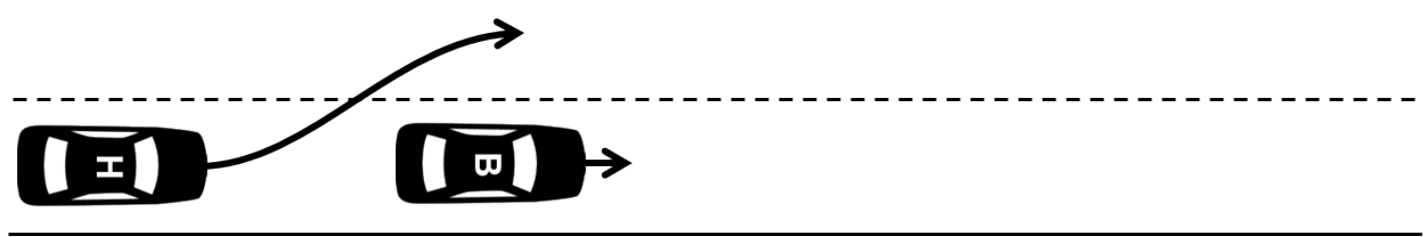

Fig. 5: Driver performed evasive steering to avoid frontal collision

Figure 5 shows the frontal collision scenario once again with the host vehicle being the following vehicle which performs an evasive steering maneuver to avoid the collision. Here, assuming the driver performs the intervention, the electric drive in combination with differential braking can be used to perform torque vectoring to enhance the responsiveness while at the same time stabilizing the vehicle. In the first part of this maneuver, it is often necessary to brake the rear inner wheel and propel the rear outer wheel to enhance the yaw response of the vehicle. However, in this extreme maneuver, typically the inner rear wheel has little to no load leading to brake interventions on the same to be ineffective. Hence the presence of an electric drive to propel the outer wheel can be very useful in this maneuver.

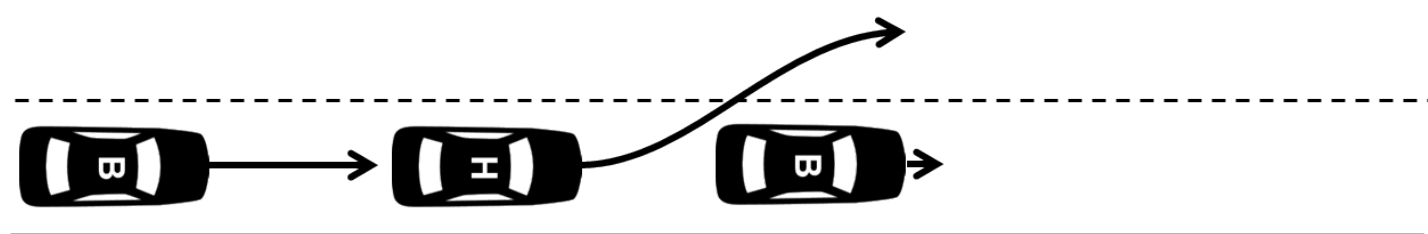

Fig. 6: Evasive steering and acceleration to avoid rear-end collision in the presence of an obstacle ahead of the host vehicle

Figure 6 shows a rear-end collision scenario case with an obstacle in front of the host vehicle as well. In this case, the driver has to accelerate and perform a lane change at the same time to avoid the accident. Due to the complexity of the maneuver, any assistance that can be provided to the driver is likely to have a significant safety impact. Differential braking alone is not likely to be helpful since it would slow the vehicle down and increase the risk of being struck from behind. IC engines are too slow to respond and hence cannot be used here. Electric drives on the other hand, in combination with differential braking can assist in both acceleration and lateral control of the vehicle in this scenario.

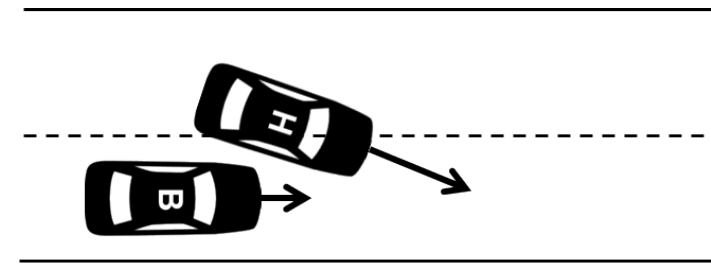

(a) Host vehicle changes lane encroaching into bullet vehicle space and is about to crash into front of bullet vehicle

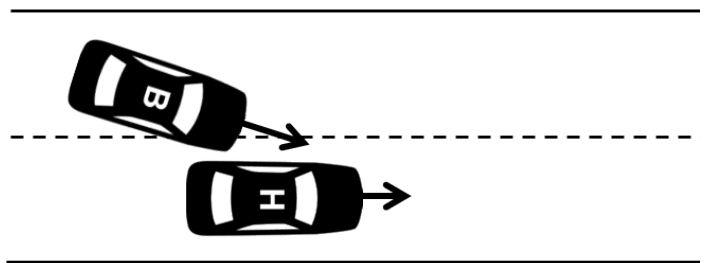

(b) Bullet vehicle changes lane enroaching into host vehicle space and is about to crash into rear of host vehicle

Fig. 7: Side swipe accidents

Acceleration using the electric drives can be used in side swipe collisions as well to improve safety. Two variants of side swipe collisions are shown in Fig. 7. In Fig. 7a, the host vehicle cuts in front of the bullet vehicle with little distance between them. In Fig. 7b, the bullet vehicle cuts in behind the host vehicle with too small a margin. In both cases, crucially, the host vehicle is ahead making acceleration the suitable 
solution. In these cases, the end goal is not necessarily to increase speed but rather to increase the margin of distance between the vehicles thereby preventing the collision. This can be done with a less severe intervention that may even not be apparent to the driver.

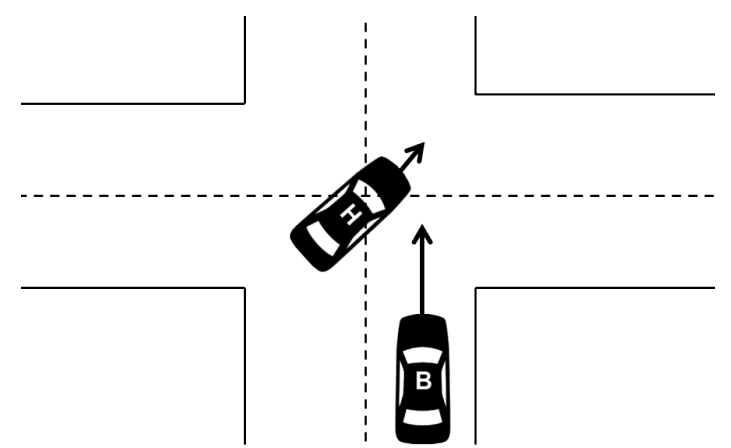

(a) Intersection accident 1

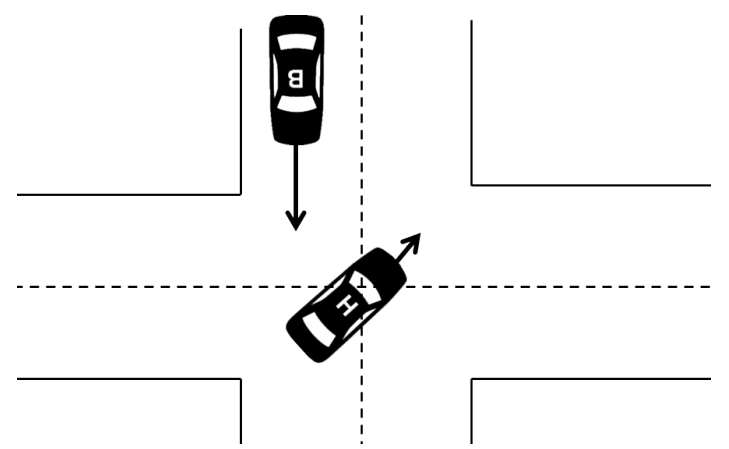

(c) Intersection accident 3

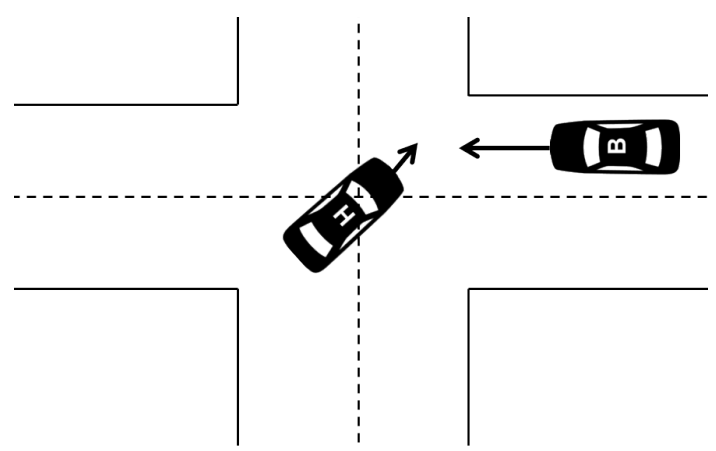

(b) Intersection accident 2

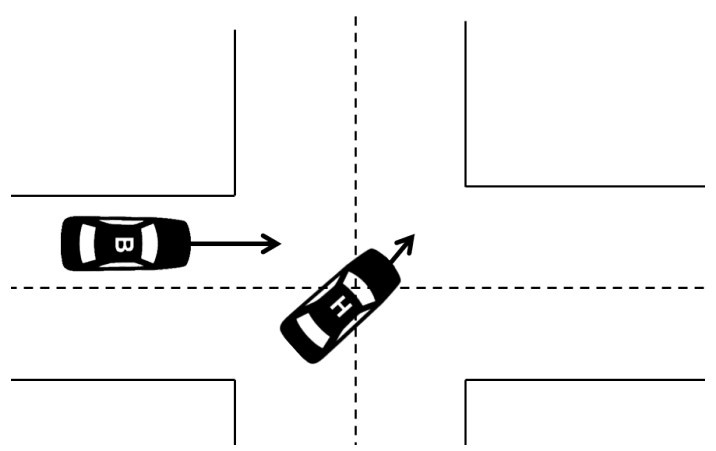

(d) Intersection accident 4

Fig. 8: Intersection accidents

A small collection of intersection accidents are shown in Fig. 8. Functionality to brake the vehicle when appropriate in the event of such collisions are already on the market. However, if the speed is high or when detection happens late or depending on the specific case, braking might no longer be a suitable option and speeding up might become necessary. In such a case, the electric drive, and perhaps differential braking to guarantee stability, might be necessary to avoid the accident.

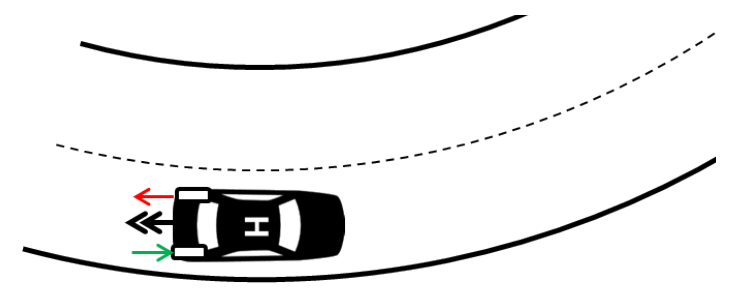

(a) Understeer control

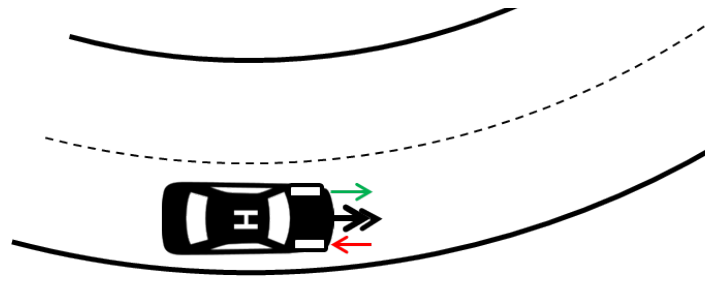

(b) Oversteer control

Fig. 9: Loss of control accidents

Two loss of control accidents (understeer and oversteer) are shown in Fig. 9. While differential braking is pretty effective in dealing with oversteer, due to the severe nature of such accidents, increased effectiveness is still welcome. Electric drives in combination with differential braking can significantly increase the yaw moment magnitude that can be applied thereby making the intervention more effective. For understeer intervention on the other hand, the ability of electric drive to propel the outer rear wheel which has the most traction can lead to large improvements in safety. Differential braking alone on the rear axle is not always very effective for understeer mitigation. 


\section{Case study: The rear-end collision scenario}

\subsection{Accelerate to mitigate or prevent being struck from behind}

With regards to being able to use electrified drivetrains for active safety interventions, the rear-end collision scenario is one of the simplest and yet most promising accident scenarios. This case is shown in Fig. 10 where the host vehicle is the lead vehicle in a rear-end collision scenario.

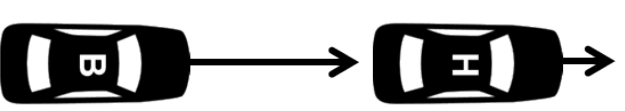

Fig. 10: Accelerate to avoid rear-end collision

The rear-end collision is one of the most accident types that occur in the world accounting for $29.7 \%$ of all accidents in the US in the year 2000. In the same year, approximately $2.2 \%$ of all licensed drivers in the US were involved in rear-end collisions and of those drivers involved in all types of crashes, $36 \%$ were involved in rear-end collisions alone [19].

While the Automatic Emergency Braking (AEB) system is already very effective in this scenario and, according to one study, can be expected to prevent and mitigate up to $35 \%$ and $53 \%$ of all such accidents respectively [20], the remaining accidents still account for a large number of accidents since this is one of the most commonly occuring accidents. These accidents could potentially be improved by a speed control intervention that accelerates the lead vehicle when a collision becomes imminent.

Such a hypothetical active safety system (henceforth termed the Automatic Emergency Acceleration system (AEA)) that moves the lead vehicle forward to prevent or mitigate a collision was envisioned and evaluated in [21]. The goal of this intervention is two-fold: first, to reduce the relative velocity between the vehicles when the collision occurs and second, to provide increased braking distance for the following vehicle if it has already started braking. The quick response time of the electric drive as compared to that of IC engines [13] is of particular benefit here. The focus of this investigation was the kinematics of the intervention and the scenario and the associated safety benefit that could be expected. Other topics such as detection, epidemiological analysis, driver interaction, etc. were discussed briefly but not analyzed in detail.

Further analysis of accident statistics pertaining to rear-end collisions shows that approximately $70 \%$ of rear-end collisions involve an impact speed of less than $30 \mathrm{~km} / \mathrm{h}$ [22], $70 \%$ have a speed difference of less than $15 \mathrm{~km} / \mathrm{h}$ [23] and between $70 \%$ to $90 \%$ involve stationary lead vehicles [24, 25]. In other words, rear-end collisions predominantly involve low speeds and that makes electric drives particularly well suited for interventions in this scenario.

Several simplifying assumptions were made to limit the scope of work. The acceleration capability of the vehicle was assumed to be limited by the grip at an axle instead of motor sizing. Given that electric motors can for brief periods supply torques much higher than their steady state rated torques, and that typically, only one of the axles is driven, a peak acceleration of $0.5 \mathrm{~g}$ was assumed. The lead vehicle was assumed to be able to reliably detect the following vehicle and also able to ensure that it is safe to accelerate in the scenario. Simplified parameterized actuator models were used consisting of an initial delay, a ramp up phase and a steady state phase were used in the analysis and simulation.

Using these assumptions, activation timings for the AEA were derived such that AEA only kicks in when it determines that collision avoidance is no longer physically possible by the following vehicle alone. Simulations were then performed using this system and the results from the same are shown in Fig. 11. 


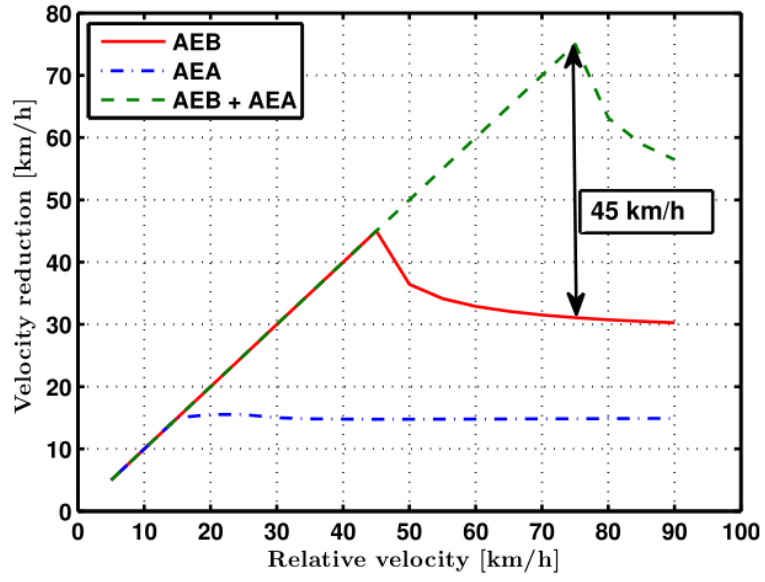

(a) Estimated velocity reduction capabilities for AEB on following vehicle, AEA on the lead vehicle and a combination of the two.

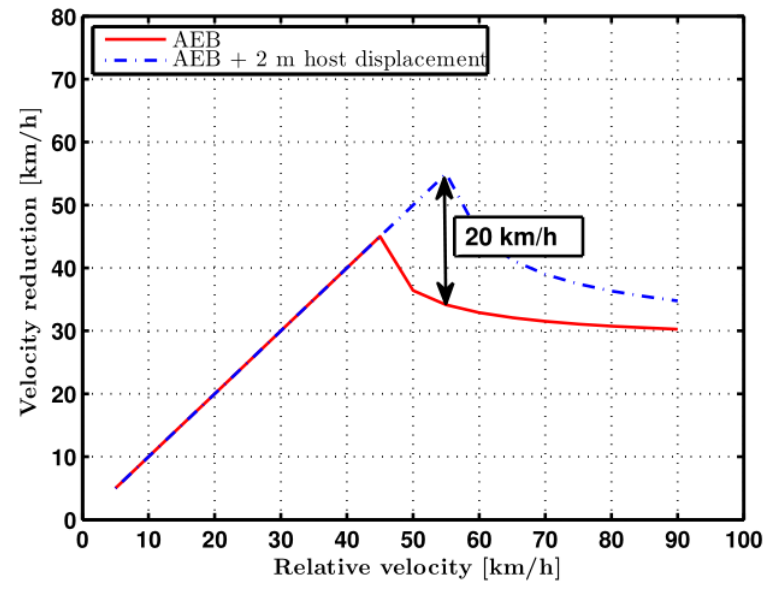

(b) Estimated velocity reduction capabilities for AEB on following vehicle, when lead vehicle moves forward by $2 \mathrm{~m}$ without a net speed increase.

Fig. 11: Velocity reduction capabilities for the AEA. From [21]

As can be seen from Fig. 11a, even with conservative activation timings, the AEA system alone can achieve velocity reductions of up to $15 \mathrm{~km} / \mathrm{h}$ when the following vehicle does not brake at all. When used in conjunction with AEB on the following vehicle, rear-end collisions with relative speeds up to $75 \mathrm{~km} / \mathrm{h}$ can be completely avoided which represents a $45 \mathrm{~km} / \mathrm{h}$ improvement over the outcome for the same case when AEB alone is used. These large velocity reductions achieved when the following vehicle brakes as well is in large part due to the fact that acceleration now not only reduces the relative speed, but also increases the braking distance available to the following vehicle.

Since just forward displacement of the lead vehicle can result in a safety benefit and since acceleration of the lead vehicle may not always be possible or advisable, a case of just moving the lead vehicle forward by a fixed distance without significantly increasing the final velocity is investigated. The expected velocity reduction from such an intervention when the lead is moved forward by $2 \mathrm{~m}$ (approximately half the length of a small car) is shown in Fig. 11b. As can be seen, up to $20 \mathrm{~km} / \mathrm{h}$ speed reduction over that of AEB alone can be achieved which represents over a $50 \%$ improvement over the AEB.

In terms of safety, the AEA system can have a large impact even with relatively small velocity reductions. The most frequently sustained injury type in rear-end crashes is the whiplash injury [26]. Several factors can influence the whiplash injury risk in these crashes, chief among them being impact severity and occupant head posture [27]. Studies on whiplash injury risk based on data from real life crashes estimate that a reduction in change of velocity of $5 \mathrm{~km} / \mathrm{h}$ for the struck vehicle can decrease the risk of sustaining whiplash symptoms lasting for more than one month by up to $65 \%$ [28]. In terms of initial symptoms the same amount of reduction in change of velocity could decrease the risk by up to $40 \%$ [28]. The potential injury risk reduction from a relatively small decrease in velocity change with AEA is therefore large.

Another potential contribution to the safety benefit could be the influence of the pre-collision acceleration on head posture. Occupant head posture with respect to the head restraint has been investigated in the past and several studies have suggested that increased distance between the head and head restraint is associated with increased risk of a whiplash injury [29, 30]. Although it remains unknown how much rearward head motion would be induced by the acceleration of the AEA system, the resulting head repositioning has the prospective safety benefit of reducing whiplash injury risk by decreasing head-to-head restraint distance before impact.

Numerous practical difficulties remain which restrict the implementation or operation of such a system. For instance, the liability involved in speeding up in a safety critical situation can be expected to be a 
major obstacle for adoption of such a system. Several options exist to allay such concerns. First is the less risky option of moving the vehicle forward instead of outright acceleration as shown in Fig. 11b. Another possibility is to use diluted versions of the AEA functions which instead of performing autonomous acceleration, could instead warn the driver and assist the driver if and when he/she tries to accelerate. This approach still takes advantage of the quick response of the electric motors but reduces liability as it offloads the decision making responsibility to the driver.

\subsection{Mitigation of secondary collision risk in evasive maneuvers for rear-end collision avoidance}

Another more sophisticated use case for improved safety using electric drives is the possiblity of speed control in evasive maneuvers to reduce the risk of secondary collisions with oncoming vehicles. This scenario has been investigated in detail in [31,32] and is illustrated in Fig. 12.

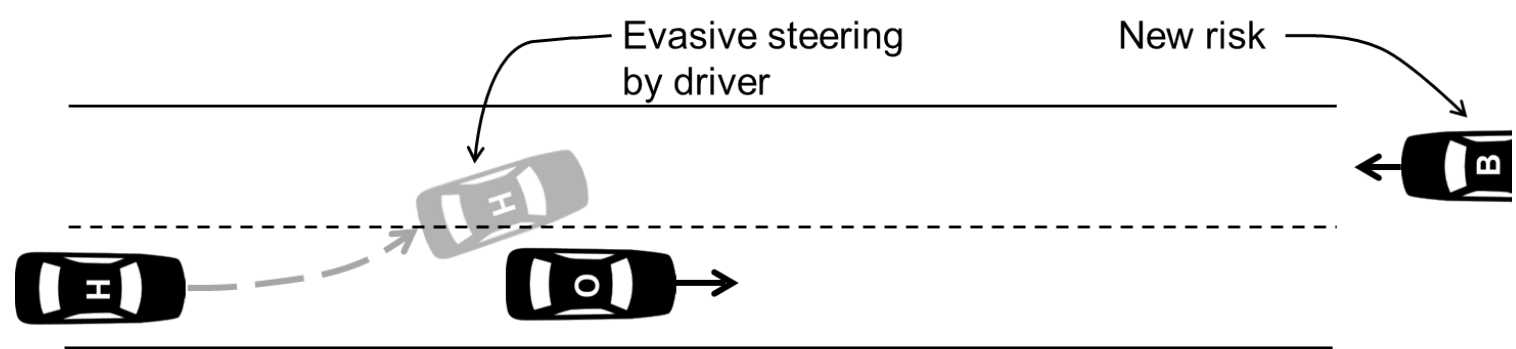

Fig. 12: Mitigation of secondary collision risk when performing evasive steering for rear-end collision avoidance

Here, the driver of the host vehicle performs an evasive steering maneuver to avoid the obstacle without any fore-knowledge of whether the adjacent lane is free. If it turns out that the adjacent lane contains a bullet vehicle, then the task of the novel safety system here would be to minimize the risk of collision with the oncoming vehicle while also assisting the driver in completing the maneuver and return to the original lane. The host vehicle is only assumed to be able to detect the oncoming vehicle after the bullet vehicle is within its line of sight partway through the maneuver.

An idealized analysis was done in [31] to better understand the maneuver kinematics and estimate the maximum safety that be achieved using electric drivetrains. It was found that under certain conditions characterized by long obstacles and large ratios of oncoming vehicle to host vehicle speeds, there is a safety benefit from maintaining speed through the maneuver. In such extreme maneuvers, typically differential braking is used to stabilize the vehicle. However, this also slows the vehicle down which as mentioned is detrimental in this case. Here, the electric drive can be used to counteract this slowdown and thereby improve safety. Additionally, an electric drive can be used to achieve torque vectoring which would be useful in stabilizing or improving the responsiveness of the vehicle through the maneuver as required.

A more realistic investigation is done in [32] wherein closed loop controllers for lateral control were designed and evaluated in simulation. Explicit speed control is not done in this case since that would require significantly more environmental information which cannot always be guaranteed to be available. Lateral control on the other hand requires much less information and can be performed in almost all cases. As such, this can be seen as a fallback solution when speed control cannot be done and needs to be very robust and reliable. The steering intervention was varied in severity in order to evaluate the performance of the controller across a range of steering interventions.

Figure 13 shows the results from the simulations with the closed loop controllers. As can be seen, three actuator sets were compared in two different scenarios; scenario A where maintaining speed is expected to have a large safety benefit and scenario B where decreasing speed is expected to be of benefit. It can be seen that in scenario A, differential brakes at best do no worse that steering and at worst make 


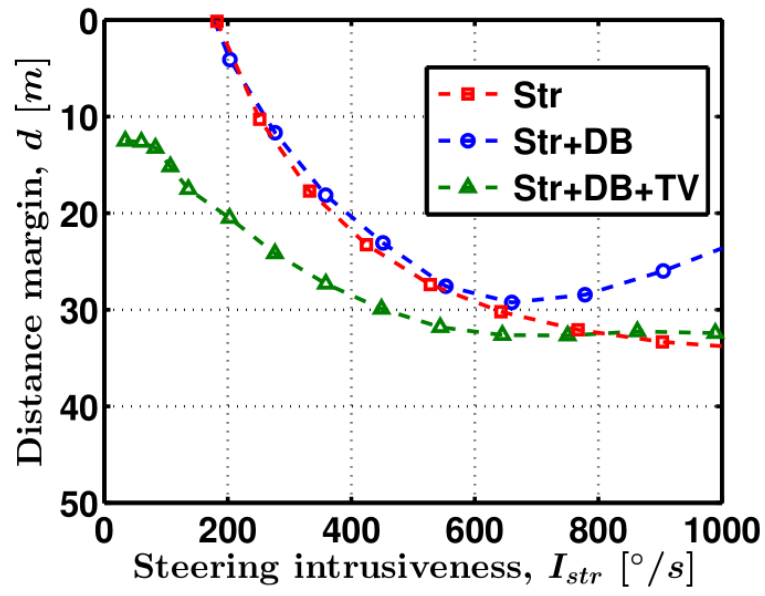

(a) Scenario A

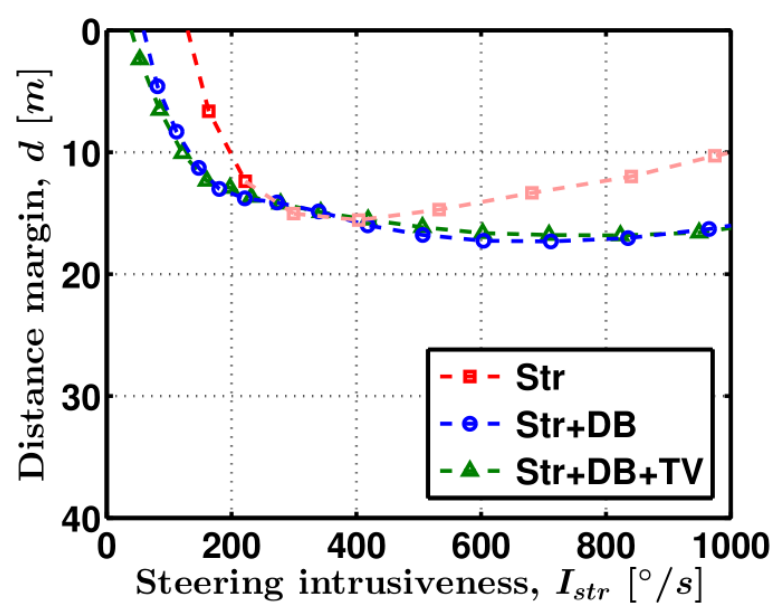

(b) Scenario B

Fig. 13: The pareto fronts from closed loop simulations for steering intrusiveness vs distance margin. In (b), the lighter plot indicates an unstable end to the maneuver. Str $=$ steering, DB $=$ Differential Braking, $\mathrm{TV}=\mathrm{Torque}$ Vectoring. From [32]

the maneuver less safe by slowing down the vehicle too much. Torque vectoring on the other hand, consistently improves safety since it can perform lateral control without slowing the vehicle down.

In scenario B, while differential braking consistently improves safety (and even prevents the vehicle from spinning out), torque vectoring does no worse and matches the performance of differential braking. This is because, while it does not slow the vehicle (which is worse in this scenario), it makes up for it by being able to supply higher yaw moment magnitudes as compared to differential braking.

Consequently, the addition of an electric drive or a dedicated torque vectoring system is seen to make lateral control interventions robust against variations in steering. Therefore, torque vectoring can act as an effective redundancy for steering which is likely to become important in modern cars with autonomous or assist functions where the driver can be out of the loop.

\section{Conclusions}

In conclusion, a need was seen for added value in electrified vehicles to make them more attractive to consumers. The possibility of achieving the same by having novel or enhanced active safety functions through the use of electric drives was investigated.

It was seen that electric drives can be used to expand the dynamic capability of the vehicle which in turn can be exploited to enhance existing or develop novel active safety functions. Several such opportunities were identified, some of which are discussed briefly in Section 3. Two specific use cases for improving safety in the rear-end collision scenario are analysed in detail in Sections 4.1 and 4.2. It was found that strong safety benefits can be achieved in both cases using electric drives.

Consequently, a strong opportunity is seen for adding safety related value to electrified vehicles at little to no extra cost.

\section{Acknowledgments}

This project was partly funded by the Fordonsstrategisk Forskning och Innovation (FFI) program of VINNOVA. Their support is gratefully acknowledged. 


\section{References}

[1] F. Chiara and M. Canova. "A review of energy consumption, management, and recovery in automotive systems, with considerations of future trends". In: Proceedings of the Institution of Mechanical Engineers, Part D: Journal of Automobile Engineering 227.6 (Mar. 25, 2013), pp. 914-936. ISSN: 0954-4070, 20412991. DOI: 10.1177/0954407012471294.

[2] M. Duvall et al. Environmental assessment of plug-in hybrid electric vehicles: Volume 1: Nationwide Greenhouse Gas Emissions. Technical report 1015325. Electric Power Research Institute, 2007.

[3] IEA. World Energy Outlook 2009. 2009. ISBN: 978-92-64-06130-9.

[4] OECD Publishing. Energy technology perspectives 2012: pathways to a clean energy system. Paris, France: OECD, 2012. ISBN: 978-92-64-17488-7.

[5] C. Tingvall. "The Zero Vision. A road transport system free from serious health losses”. In: Transportation, traffic safety and health (1997), pp. 37-57.

[6] J. Whitelegg and G. Haq. "Vision zero: Adopting a target of zero for road traffic fatalities and serious injuries". In: Stockholm Environment Institute (2006).

[7] Vision Zero: Seattles plan to end traffic deaths and serious injuries by 2030 - VisionZero | seattle.gov. 2015. URL: http: //www . seattle.gov/visionzero (visited on 04/20/2015).

[8] Zero Traffic Deaths In San Francisco by 2024. 2015. uRL: http : / / visionzerosf . org/ (visited on 04/20/2015).

[9] Vision Zero|New York City. 2015. uRL: http://www .nyc.gov/html/visionzero/pages/home/home. shtml (visited on 04/20/2015).

[10] Vision Zero | The City of Portland, Oregon. 2015. urL: https : / / www . portlandoregon . gov / transportation/66612 (visited on 04/20/2015).

[11] G. Jost et al. "Road safety target outcome: 100.000 fewer deaths since 2001. 5th Road Safety PIN Report". In: Brussels: European Transport Safety Council (2011).

[12] Statistics accidents data - European Commission. Data source: CARE (EU road accidents database). 2015. URL: http : //ec.europa.eu/transport/road_safety/specialist/statistics/index_en.htm (visited on 05/07/2015).

[13] Y. Hori, Y. Toyoda, and Y. Tsuruoka. "Traction control of electric vehicle based on the estimation of road surface condition-basic experimental results using the test EV UOT Electric March". In: Power Conversion Conference-Nagaoka 1997., Proceedings of the. Vol. 1. 1997, pp. 1-8.

[14] S. Murata. "Innovation by in-wheel-motor drive unit". In: Vehicle System Dynamics 50.6 (June 1, 2012), pp. 807-830. ISSN: 0042-3114. DOI: 10.1080/00423114.2012.666354.

[15] A. Arikere. "New Vehicle Functionality Using Electric Propulsion”. Master Thesis. Göteborg: Chalmers University of Technology, 2012.

[16] A. Arikere. "Vehicle Dynamic Opportunities in Electrified Vehicles for Active Safety Interventions". Licentiate thesis. Göteborg: Chalmers University of Technology, 2015.

[17] E. Coelingh, A. Eidehall, and M. Bengtsson. "Collision Warning with Full Auto Brake and Pedestrian Detection - a practical example of Automatic Emergency Braking”. In: 2010 13th International IEEE Conference on Intelligent Transportation Systems (ITSC). 2010 13th International IEEE Conference on Intelligent Transportation Systems (ITSC). Sept. 2010, pp. 155-160. DOI: 10. 1109/ITSC. 2010. 5625077.

[18] C. Gauss. Comparative test of advanced emergency braking systems. Test report. ADAC Technik Zentrum, Landsberg am Lech, Germany, Aug. 30, 2012.

[19] S. Singh. Driver attributes and rear-end crash involvement propensity. Technical report DOT HS 809540. NHTSA, 2003.

[20] H. Schittenhelm. "Advanced Brake AssistReal World effectiveness of current implementations and next generation enlargements by Mercedes-Benz". In: Proceedings of the 23rd International Technical Conference on the Enhanced Safety of Vehicles (ESV). Enhanced Safety of Vehicles (ESV). Seoul, South Korea, May 27, 2013. 
[21] A. Arikere et al. "On the Potential of Accelerating an Electrified Lead Vehicle to Mitigate Rear-End Collisions". In: Proceedings of the 3rd International Symposium on Future Active Safety Technology Towards zero traffic accidents, 2015. FAST-zero 15. Göteborg, Sweden, Sept. 9, 2015, pp. 377-384.

[22] V. Eis, R. Sferco, and P. A. Fay. "A detailed analysis of the characteristics of European rear impacts". In: Proceedings: International Technical Conference on the Enhanced Safety of Vehicles. Vol. 2005. National Highway Traffic Safety Administration, 2005, 9p-9p.

[23] W. Hell et al. "Consequences for seat design due to rear end accident analysis, sled tests and possible test criteria for reducing cervical spine injuries after rear-end collision". In: Proceedings of the International Research Council on the Biomechanics of Injury conference. Vol. 27. International Research Council on Biomechanics of Injury, 1999, pp. 243-259.

[24] K. Kodaka et al. "Rear-end collision avoidance assist system". In: Signal (1997).

[25] R. R. Knipling, J.-S. Wang, and H.-M. Yin. Rear-end Crashes: Problem Size Assessment and Statistical Decsription. National Highway Traffic Safety Administration, 1993.

[26] Y. Watanabe et al. "Influence of seat characteristics on occupant motion in low-speed rear impacts". In: Accident Analysis \& Prevention 32.2 (Mar. 2000), pp. 243-250. IssN: 0001-4575. Dor: 10. 1016/S00014575(99)00082-2.

[27] A. Carlsson. "Addressing Female Whiplash Injury Protection - A Step Towards 50th Percentile Female Rear Impact Occupant Models". Doctoral thesis. Chalmers University of Technology, 2012.

[28] M. Krafft et al. "Influence of crash severity on various whiplash injury symptoms: A study based on real-life rear-end crashes with recorded crash pulses". In: Proceedings 19th ESV Conf. Vol. 5. 2005, p. 0363.

[29] C. M. Farmer, J. K. Wells, and J. V. Werner. "Relationship of head restraint positioning to driver neck injury in rear-end crashes". In: Accident Analysis \& Prevention 31.6 (Nov. 1999), pp. 719-728. IssN: 0001-4575. DOI: $10.1016 / 50001-4575(99) 00035-4$.

[30] L. Jakobsson. "Field Analysis of AIS1 Neck Injuries in Rear-End Car Impacts". In: Journal of Whiplash \& Related Disorders 3.2 (Jan. 1, 2004), pp. 37-53. Issn: 1533-2888. Dor: 10.3109/J180v03n02\_04.

[31] A. Arikere et al. "The Potential Safety Benefit of Propulsion in Obstacle Avoidance Manoeuvres with Oncoming Traffic". In: Proceedings of the 12th International Symposium on Advanced Vehicle Control. AVEC '14. Tokyo, Japan, Sept. 22, 2014, pp. 126-131.

[32] A. Arikere, M. Lidberg, and G. Olsson. "The trade-off between distance margin and steering effort in obstacle avoidance manoeuvres with oncoming traffic". In: Proceedings of the 24th International Symposium on Dynamics of Vehicles on Roads and Tracks. IAVSD 2015. Graz, Austria, Aug. 17, 2015.

\section{Authors}

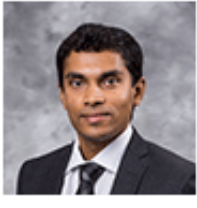

Adithya Arikere is currently employed as a vehicle dynamics and controls engineer at the Trollhättan Technical Center (TTC) of AAM (American Axle \& Manufacturing), Sweden. $\mathrm{He}$ is also working on his $\mathrm{PhD}$ degree in vehicle dynamics at Chalmers University of Technology, Gothenburg as part of a project funded by AAM and Fordonsstrategisk Forskning och Innovation (FFI).

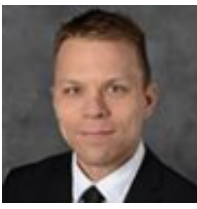

Anders Tysk is the Director of Business Development at AAM Europe. He holds a Master degree in civil engineering from University of Lulea. He has over 20 years of global experience in the automotive industry and has previously worked at Saab and GM. He has spearheaded the development of electric propulsion and torque vectoring drives at Saab and AAM and has overseen several advanced engineering activities and program launches. 